\title{
Recent highlights from the PHENIX heavy ion program
}

\author{
J.C.Hill on behalf of the PHENIX Collaboration ${ }^{1, a}$ \\ ${ }^{1}$ lowa State University
}

\begin{abstract}
It is accepted that a QGP can be formed in relativistic collisions of heavy nuclei $(\mathrm{A}+\mathrm{A})$. Recently long-range correlations have been observed in $\mathrm{p}+\mathrm{A}$ collisions at the LHC in high multiplicity events. PHENIX has carried out a series of studies of $\mathrm{d}+\mathrm{Au}$ collisions at $200 \mathrm{GeV}$ to see if such correlations persist at lower energies compared to those at the LHC. Results of a study of long-range correlations and flow are presented for $\mathrm{d}+\mathrm{Au}$ collisions. Data from $\mathrm{Au}+\mathrm{Au}$ collisions collected during the beam energy scan (BES) was used to determine both quark and nucleon number scaling. The HBT method was used to determine radii of the fireball at kinetic freezeout. Implications for the nuclear EOS are discussed. Also results of a search for "dark photons" are presented. Recent PHENIX highlights on heavy flavor, electromagnetic probes, spin and plans for PHENIX upgrades were presented in other talks at this conference.
\end{abstract}

\section{Introduction}

There are many recent highlights from the PHENIX Heavy Ion Program. In this paper I will discuss recent results from $\mathrm{d}+\mathrm{Au}$ collision studies, the beam energy scan (BES) and the search for dark photons. In addition at this conference recent PHENIX results on probing the properties of hot dense QCD matter with heavy flavor were discussed by Rachid Nouicer and results from direct photon measurements were discussed by Ilia Ravinovich. A discussion of possible future measurements at PHENIX using the planned upgrade to sPHENIX was given by Eric Mannel. In addition there is a nuclear spin program at PHENIX and recent highlights from that program was discussed by Kiyoshi Tanida.

\section{Status of our knowledge of hot and dense QCD matter}

Studies of the matter produced in ultrarelativistic heavy ion collisions provide a method for studying the QCD phase diagram[1]. A typical phase diagram for nuclear matter is shown in figure 1. From studies of the hot and dense QCD matter created in relativistic heavy ion collisions over a period of years a number of conclusions can be reached.

1. A hot dense medium is created in $\mathrm{Au}+\mathrm{Au}$ collisions. Evidence is observation of nuclear modification factors $R_{A A}<1.0$.

a e-mail: jhill@iastate.edu 
2. The hot dense medium flows with low viscosity. Evidence is $v_{2}>0$ and observation of the ridge.

3. A quark-gluon plasma (QGP) is actually created. Evidence for this is quark scaling of $v_{2}>0$ for mesons $(\mathrm{q}=2)$ and baryons $(\mathrm{q}=3)$.

4. The lack of observation of a phase transition implies crossover from a QGP to the hadron gas phase.

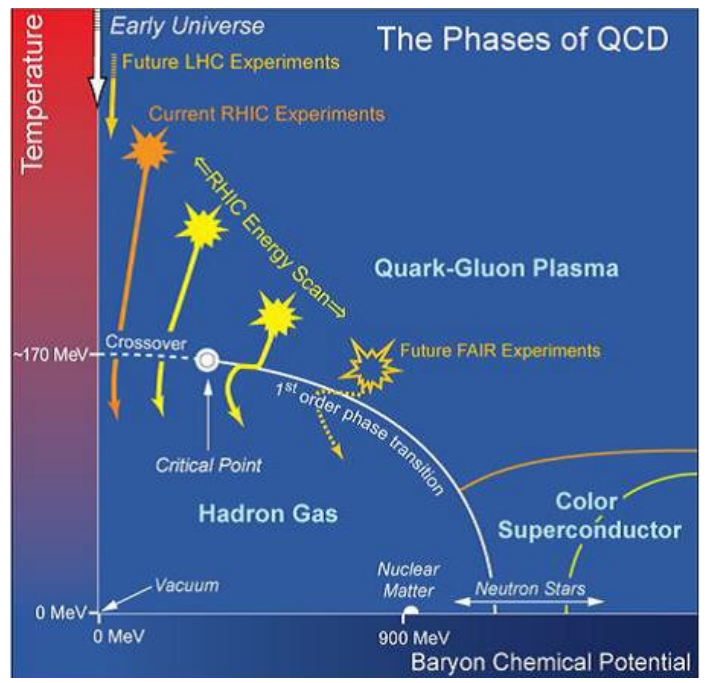

Figure 1. Possible phase diagram for nuclear matter

A critical aspect of the establishment of the nature of the QGP has been the observation that the hot dense matter created in relativistic heavy ion collisions flows. The geometry of flow is illustrated in figure 2.

In studying flow the following points are relevant.

1. The reaction geometry produces an almond shaped interaction region.

2. Compression of mass in the center produces an anisotropic $p_{T}$ distribution.

3. The resulting $p_{T}$ distribution is described in terms of $\left[1+\sum_{n=1}^{\infty} 2 v_{2} \cos \left[n\left(\phi-\Psi_{R}\right]\right]\right.$

4. A finite $v_{2}$ is termed elliptic flow. $\Psi$ is in the plane of the beam and the impact parameter.

Flow data [2] for $v_{2}$ for $200 \mathrm{GeV} / \mathrm{A}$ collisions of $\mathrm{Au}+\mathrm{Au}$ from both STAR and PHENIX is shown in figure 3. The low values of $v_{2}$ show that flow is a prominent feature of $\mathrm{Au}+\mathrm{Au}$ collisions. Relativistic hydrodynamic calculations [3] are a good fit to the data for $p_{T}<2.0 \mathrm{Gev} / \mathrm{c}$.

\section{Possible long range correlations and flow in small systems}

In relativistic A-A collisions a QGP medium is formed which signals its presence through long range correlations and finite flow $v_{2}$. It was thought that $\mathrm{p}+\mathrm{p}$ and $\mathrm{p}+\mathrm{A}$ collisions could not form such a 


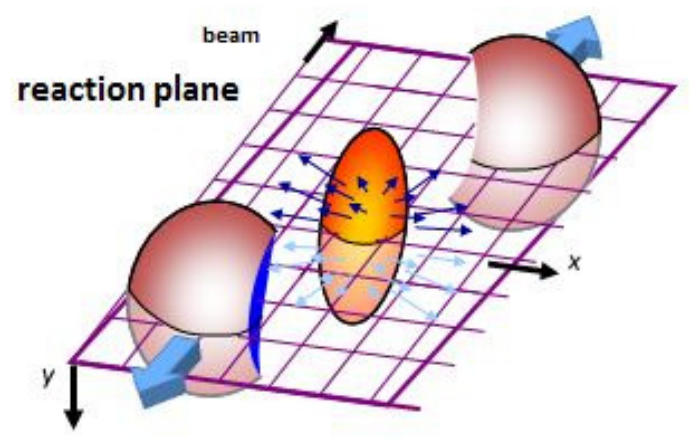

Figure 2. Diagram showing the geometry of flow for collisions of heavy nuclei

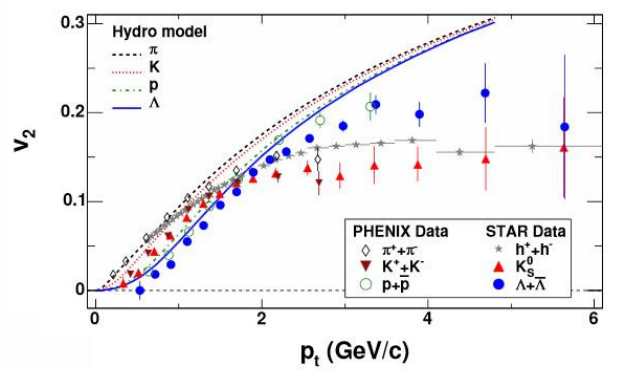

Figure 3. Data for $v_{2}$ for $200 \mathrm{GeV} / \mathrm{A}$ collisions of $\mathrm{Au}+\mathrm{Au}$.

medium because of the small system size. Recently results from ALICE [4] and CMS [5] from 5.02 $\mathrm{TeV} \mathrm{p}+\mathrm{Pb}$ collisions at the LHC indicate the presence of long range correlations. This is shown in figure 4 where both a small $v_{2}$ and a ridge were observed, both indicating long range correlations and significant flow. One might argue that for the lower beam energies at RHIC such correlations would be absent or much smaller. It is thus of interest to explore whether a small $v_{2}$ and a ridge would be observed with $\mathrm{d}+\mathrm{Au}$ collisions at RHIC at $200 \mathrm{GeV} / \mathrm{A}$.
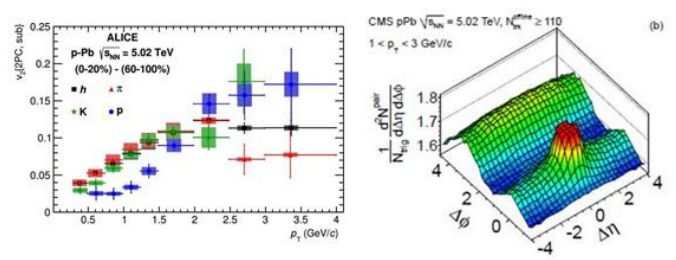

Figure 4. Flow and the ridge for $5.02 \mathrm{TeV} \mathrm{p}+\mathrm{Pb}$ collisions at the $\mathrm{LHC}$ 
In figure 5 results for $v_{2}$ for $200 \mathrm{GeV} \mathrm{d}+\mathrm{Au}$ for the 0 to $5 \%$ most central collisions are shown [6] and compared with results of similar centrality for $5.02 \mathrm{TeV} \mathrm{p}+\mathrm{Pb}$ collisions at the LHC [4]. The following observations can be made.

1. Mass splitting of $v_{2}$ is seen for both $\mathrm{d}+\mathrm{Au}$ and $\mathrm{p}+\mathrm{Pb}$ reactions with $v_{2}$ generally larger for pions.

2. Viscous hydrodynamics [7] describes $\mathrm{d}+\mathrm{Au}$ below $p_{T}=2.0 \mathrm{GeV} / \mathrm{c}$.

3. Note larger mass splitting for $\mathrm{p}+\mathrm{Pb}$ below $p_{T}=2.0 \mathrm{GeV} / \mathrm{c}$ that may indicate stronger radial flow for $\mathrm{p}+\mathrm{Pb}$.

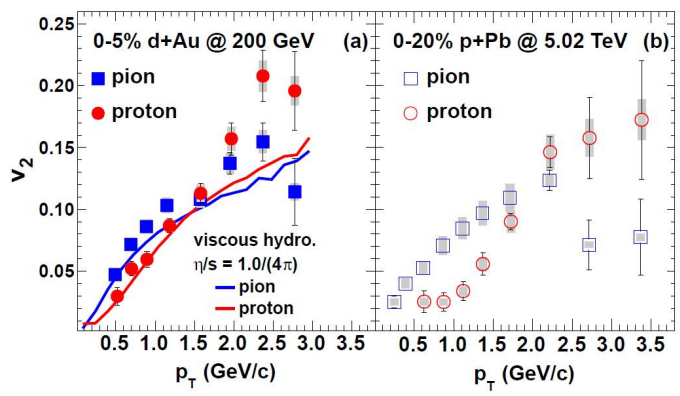

Figure 5. $v_{2}$ observed for $200 \mathrm{GeV} \mathrm{d}+\mathrm{Au}$ collisions

It is clear from figure 5 that a non-zero $v_{2}$ and flow are observed for both relativistic $\mathrm{d}+\mathrm{Au}$ collisions at RHIC and $\mathrm{p}+\mathrm{Pb}$ collisions at the LHC. It is thus of interest to determine if a ridge is observed in $\mathrm{d}+\mathrm{Au}$ collisions at RHIC. Therefore a correlation function $C\left(\Delta \phi, p_{T}\right)$ is constructed in the following steps.

1. Correlate one track in the central arm with one in the forward muon piston calorimeter (MPC).

2. Construct a signal distribution $S\left(\Delta \phi, p_{T}\right)$ where $\phi=\phi_{\text {track }} \breve{\phi_{\text {tower }} \text {. }}$

3. Construct mixed-event distribution $M\left(\Delta \phi, p_{T}\right)$ from different events.

4. Construct a normalized correlation function $C\left(\Delta \phi, p_{T}\right)$.

Correlation functions for central $\mathrm{d}+\mathrm{Au}$ collisions and mid-bias $\mathrm{p}+\mathrm{p}$ collisions for several $p_{T}$ ranges [6] are shown in figure 6. Fits from $\mathrm{c} 1$ to $\mathrm{c} 4$ in $\cos (n \Delta \phi)$ are shown. The following conclusions can be drawn.

1. The $\mathrm{p}+\mathrm{p}$ reactions are dominated by the dipole term (no ridge).

2. The $d+A u$ reactions show a near side peak (ridge) that increases with $p_{T}$.

Since $d+A u$ reactions show a ridge it is of interest to study in more detail the separate correlation functions from the $\mathrm{d}$ going and the Au going sides of the reaction. The result of this comparison is shown in figure 7. Note that a ridge is clearly visible for the Au but not for the d going side. Also the $c_{2}$ component for the $\mathrm{d}$ going side is not zero but is much reduced compared to $c_{2}$ for the $\mathrm{Au}$ going side. Studies of the correlation functions have also been made for the $\mathrm{d}$ and $\mathrm{Au}$ going sides as a function of centrality. For the Au going side there is a clear ridge that emerges as centrality is 

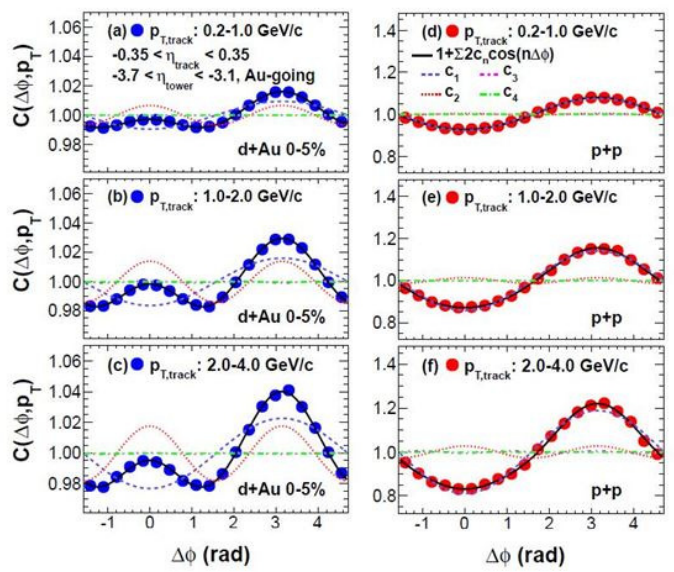

Figure 6. Correlation functions for $\mathrm{Au}+\mathrm{Au}$ and $\mathrm{p}+\mathrm{p}$ collisions at RHIC

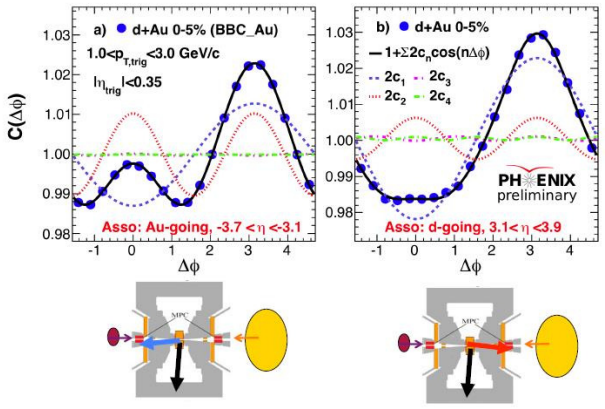

Figure 7. Comparison of correlation functions for the $\mathrm{d}$ going and Au going sides of the $\mathrm{d}+\mathrm{Au}$ collisions

increased. The peripheral collision pattern is similar for the $\mathrm{d}$ and Au sides showing essentially no ridge. For the d side no ridge is observed but the $c_{2}$ correlation increases with centrality [8].

During Run-14 in the spring of $2014 \mathrm{He}^{3}+\mathrm{Au}$ collision data was takenfor two weeks at RHIC. A data sample of 2.2 B events at mid-bias (MB) was collected. We are eager to see if the odd geometry of $\mathrm{He}^{3}$ gives us a significant $n_{3}$ component and more flow.

\section{Beam energy scaling results}

Since the first collisions of $\mathrm{Au}+\mathrm{Au}$ in the summer of 2000, RHIC has run a number of energy projectile combinations including $\mathrm{Au}+\mathrm{Au}, \mathrm{U}+\mathrm{U}, \mathrm{Cu}+\mathrm{Au}, \mathrm{Cu}+\mathrm{Cu}, H e^{3}+\mathrm{Au}, \mathrm{d}+\mathrm{Au}$ and $\mathrm{p}+\mathrm{p}$. The results from $\mathrm{Au}+\mathrm{Au}$ collisions can be used to test beam energy scaling. RHIC has provided $\mathrm{Au}+\mathrm{Au}$ collision energies of $7.7,15,19.6,27,39,62.4,130$ and $200 \mathrm{GeV}$ providing a wide range of energies to test energy scaling. To test quark scaling yields of $\mathrm{Au}+\mathrm{Au}$ collisions from 7.7 to $200 \mathrm{GeV}$ were determined as a function of centrality [9]. The results are shown in figure 8. The plot on the left in the figure shows the yield of $\mathrm{Au}+\mathrm{Au}$ collisions from 7.7 to $200 \mathrm{GeV}$ as a function of centrality but 
divided by the number of valence quarks. The plot on the right shows the same data but with the highest centrality points for each beam energy normalized to 1.0 to show trends.
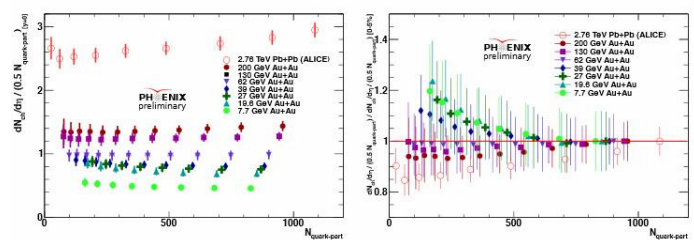

Figure 8. Beam energy scan quark scaling results for $\mathrm{Au}+\mathrm{Au}$ collisions

As can be seen from figure 8 quark scaling works well from 200 to $62 \mathrm{GeV}$ but breaks down at lower energies. A plot can also be made (not shown) [9] where the yield is divided by the number of nucleons rather than the number of quarks. That plot shows that nucleon scaling works well for energies below $40 \mathrm{GeV}$.

\section{Studies of nuclear fireball radii using the HBT method}

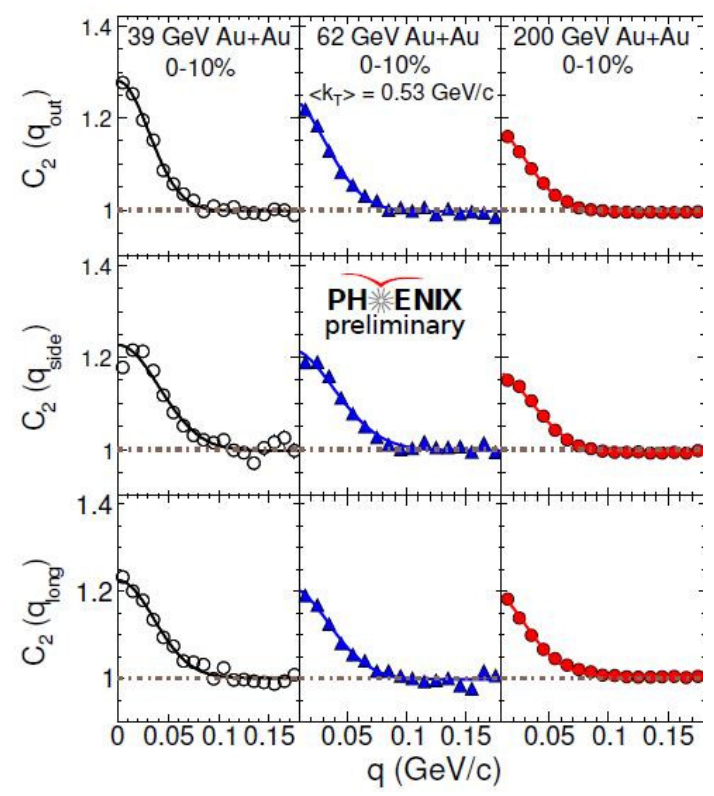

Figure 9. $C_{2}$ for 39,62 and $200 \mathrm{GeV} \mathrm{Au}+\mathrm{Au}$ central reactions

In 1956 Hanbury Brown and Twiss (HBT) measured the angular diameter of Sirius from light by observing correlations of light from different parts of the planet's surface [10]. In 1960 Goldhaber et al. measured correlation functions between pions in $\mathrm{p}+$ pbar reactions [11]. It is thus possible to use HBT to determine correlation functions for the nuclear fireball at kinetic freeze out. In order to do 
this 2-particle pion correlation functions of the form $C_{2}(q)=A(q) / B(q)$ were constructed using the following steps.

1. $\mathrm{A}(\mathrm{q})$ is the measured distribution momentum difference $\mathrm{q}=p_{2}-p_{1}$.

2. $B(q)$ is the pair uncorrelated distribution from different events.

3. $C_{2}(q)=N\left[(\lambda(1+G(q))) F_{c}+(\lambda-l)\right]$

4. $\mathrm{G}(\mathrm{q})=\exp \left(-R_{\text {side }}^{2} q_{\text {side }}^{2}-R_{\text {out }}^{2} q_{\text {out }}^{2}-R_{\text {long }}^{2} q_{\text {long }}^{2}\right)$

In the equations above $\mathrm{N}=$ normalization factor, $\lambda=$ correlation strength, $F_{c}=$ Coulomb correction factor and the R's are the measured Gaussian HBT radii. The parameterization of Bertsch[12] and Pratt[13] was used for $\mathrm{R}$ where $R_{\text {long }}$ is measured in the $q_{\text {long }}=0$ frame. The parameter $q_{\text {long }}$ is along the beam direction, $q_{\text {out }}$ is parallel to $k_{T}$ of the pair and $q_{\text {side }}$ is perpendicular to the beam and $k_{T}$ of the pair. The measured $C_{2}(q)$ can be used to determine R.

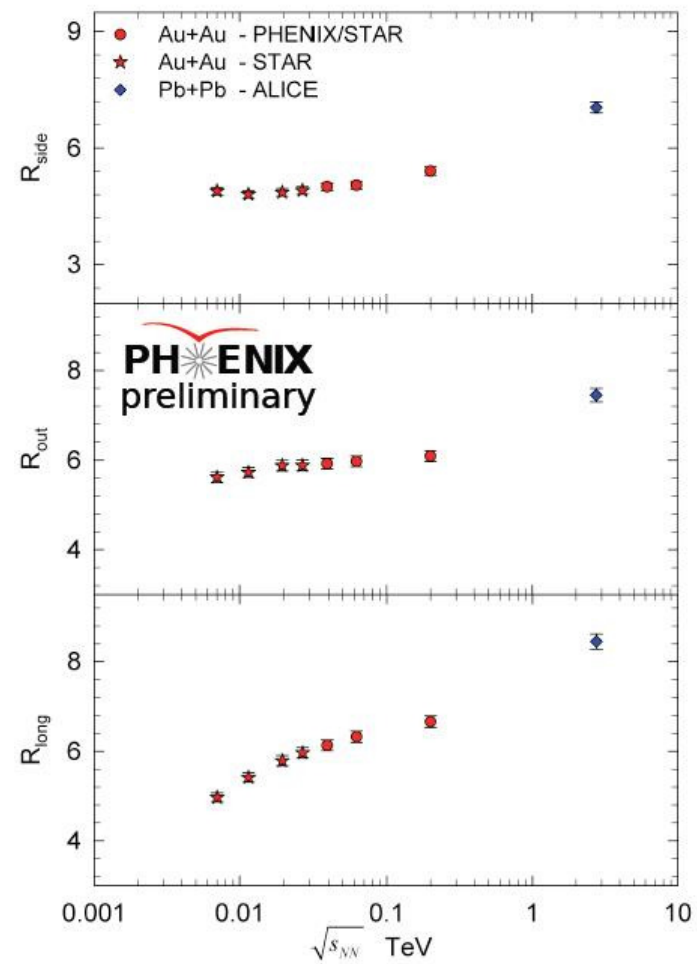

Figure 10. Results for HBT radii vs collision energy

The $C_{2}$ correlation functions for 39, 62 and $200 \mathrm{GeV}$ collisions of $\mathrm{Au}+\mathrm{Au}$ [14] are shown in figure 9 . From the correlation functions $C_{2}\left(q_{\text {out }}\right), C_{2}\left(q_{\text {side }}\right)$ and $C_{2}\left(q_{\text {long }}\right)$ the corresponding HBT pion radii $R_{\text {out }}, R_{\text {side }}$ and $R_{\text {long }}$ were calculated [9]. The radii have interesting scaling properties. Results for radii determined by both STAR[15] and PHENIX [14] are consistent with radii scaling linearly with 
$m_{T}^{-1 / 2}$. In addition it is found that the HBT pion radii scale linearly with the initial radius [14]. These results are consistent with calculations [16] which associate a larger expansion time with a larger size.

In figure 10 the measured HBT pion radii are plotted as a function of $\mathrm{Au}+\mathrm{Au}$ collision energy [14]. The results include data from PHENIX, STAR and ALICE. All the data is interpolated to $m_{T}=$ $0.26 \mathrm{GeV}$. This is valid due to $m_{T}$ scaling. For all three radii an increase of kinetic freeze out radius with collision energy is observed.

Ratios and differences of various HBT radii give information on both the kinetic freeze out time $\tau$ and the duration of kinetic freeze out $\Delta \tau . R_{\text {out }}^{2}-R_{\text {side }}^{2}$ is a proxy for emission duration $\Delta \tau$ and $R_{\text {side }} / R_{\text {long }}$ is a proxy for expansion speed and the speed of sound $c_{s}$ in the medium [16]. The results for these parameters as a function of $\mathrm{Au}+\mathrm{Au}$ collision energy are shown in figure 11. The results are not linear with energy. The curve for $R_{\text {out }}^{2}-R_{\text {side }}^{2}$ shows a maximum in the vicinity of $30 \mathrm{GeV}$ collision energy. The curve for $R_{\text {side }} / R_{\text {long }}$ shows a minimum in the vicinity of $30 \mathrm{GeV}$ [14]. These nonmonotonic patterns are consistent with the minimum observed as a function of collision energy for the viscous coefficients [17] and could be a further indication of trajectories passing through the softest region in the Equation of State (EOS).

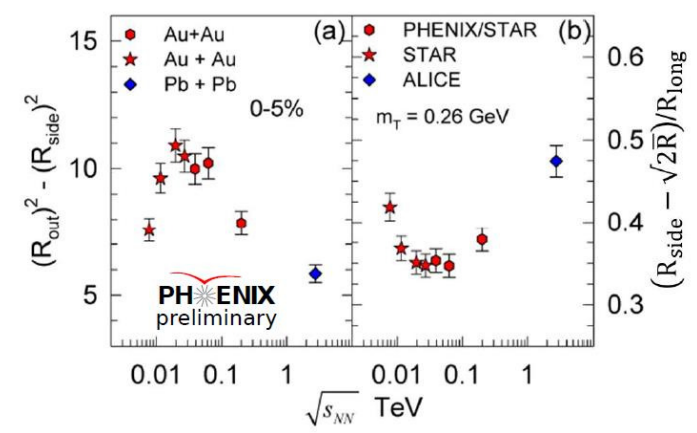

Figure 11. Trends of radii results with energy

\section{Dark photons and the muon g-2 standard model discrepancy}

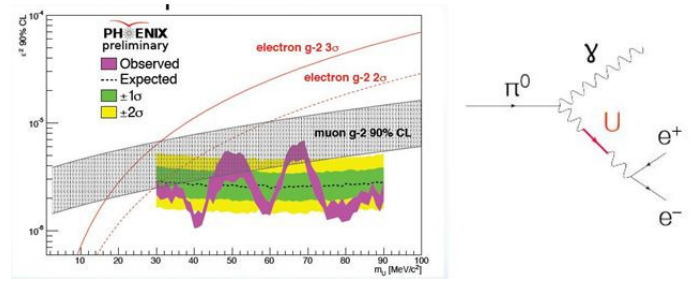

Figure 12. Dark photons and the muon g-2 standard model discrepancy

Precise experiments to determine the muon g-2 parameter have measured a 3.6 $\sigma$ discrepancy with predictions from the standard model (SM) [18]. The introduction of a new particle called a dark 
photon U has been introduced [19] to explain the discrepancy. Many experiments have been carried out that limit the U mass range. Even so a muon g-2 explainable band (90\% CL) still survives for $30-50 \mathrm{MeV} / c^{2} \mathrm{U}$ mass. A possible decay pattern for the decay of a $\pi^{0}$ to a photon and a $\mathrm{U}$ particle with the $\mathrm{U}$ decaying to a Dalitz pair is shown in figure 12. PHENIX has a large sample of $\pi^{0} \mathrm{~s}$ and a long history of $e^{+} e^{-}$analysis. The $\pi^{0} / \eta>\gamma U>\gamma e^{+} e^{-}$reaction was measured using 1.4 million Dalitz pairs from $p+p$ and $d+A u$ collisions. The resulting $90 \%$ CL results are shown in figure 12 . In more recent PHENIX measurements the region from 36 to $90 \mathrm{MeV} / c^{2}$ was eliminated [20] but a small region still survives from 30 to $36 \mathrm{MeV} / \mathrm{c}^{2}$ as seen from figure 12 .

\section{Conclusions}

1. Evidence for long range correlations and flow was observed for $200 \mathrm{GeV} \mathrm{d}+\mathrm{Au} v_{2}$ and ridge measurements at RHIC.

2. The RHIC beam energy scan (BES) shows break down in quark scaling below $62 \mathrm{GeV}$. Nucleon scaling works well below $40 \mathrm{GeV}$.

3. HBT measurements have determined fireball radii for $\mathrm{Au}+\mathrm{Au}$ collisions at kinetic freeze out which suggests possible softening of the nuclear matter equation of state in the vicinity of 30 GeV.

4. Dalitz electron pair measurements have limited the range of dark-photon masses as an explanation of the muon g-2 anomaly.

\section{References}

[1] M. A. Stephanov, K. Rajagopal, and E. V. Shuryak, Phys. Rev. Lett. 81, 4816 (1998)

[2] J. Adams et al., Phys. Rev. C 72, 014904 (2005)

[3] P. Huovinen, private communication to STAR (2004)

[4] B. Abelev et al. (ALICE Collaboration), Phys. Lett. B726, 164 (2013)

[5] D. Abbaneo et al. (CMS Collaboration), Phys. Lett. B718, 795 (2013)

[6] A. Adare et al. (PHENIX Collaboration), arXiv:1404.7461 (2014)

[7] P. Romatschke, private communication (2014)

[8] N.N. Ajitanand for the PHENIX Collaboration, Quark Matter 2014 Proceedings

[9] R. Soltz for the PHENIX Collaboration, Quark Matter 2014 Proceedings

[10] R. H. Brown and R. Q. Twiss, Nature 178, 1046 (1956)

[11] G. Goldhaber, S. Goldhaber, W. Lee, and A. Pais, Phys. Rev.120, 300 (1960)

[12] G. Bertsch, Nucl. Phys. A498,173C (1989)

[13] S. Pratt, Phys. Rev. D 33, 1314 (1986)

[14] A. Adare et al. (PHENIX Collaboration), arXiv:1410.2559 (2014)

[15] L. Adamczyk et al. (STAR Collaboration), arXiv:1403.4972

[16] E. Shuryak and I. Zahed, Phys. Rev. C 88, 044915 (2013).

[17] R. A. Lacey, A. Taranenko, J. Jia, D. Reynolds, N. N. Ajitanand, J. M. Alexander, Y. Gu, and A.

Mwai, Phys. Rev. Lett. 112, 082302 (2014)

[18] J. Beringer et al., Phys. Rev. D86, 010001 (2012)

[19] H. Davoudiasl, H.-S. Lee, W. J. Marciano, Phys. Rev. D86, 095009 (2012)

[20] A. Adare et al. (PHENIX Collaboration), arXiv:1409.0851 (2014) 